\title{
VENEI
}

Encontro Nacional de Economia Industrial e Inovação

\section{A influência das dimensões de proximidades na decisão de colaboração científica brasileira}

\author{
Daniel Oliveira Costa (Mestrando em Economia aplicada pelo PPGE/FE- Universidade Federal de Juiz de Fora); \\ Eduardo Gonçalves (Professor da Faculdade de Economia da Universidade Federal de Juiz de Fora e do PPGE); \\ Renato de Castro Garcia (Professor da Faculdade de Economia da Universidade Estadual de Campinas);
}

Resumo:

O objetivo deste trabalho é entender a influência da proximidade social, institucional, organizacional, cognitiva e geográfica no contexto colaborativo entre pesquisadores no Sistema Nacional de Inovação (SNI) brasileiro no período entre 2009 a 2017. Trabalhos empíricos discutem essas proximidades em países com SNI desenvolvidos, servindo de referência para o presente estudo no SNI brasileiro, o qual é considerado imaturo. Embora os trabalhos sobre proximidades sejam vastos, a maioria desses estudos não consideram todas essas dimensões simultaneamente. Para construção da rede de pesquisadores e extração das proximidades foram utilizados dados extraídos da plataforma Lattes do Conselho Nacional de Desenvolvimento Científico e Tecnológico (CNPq). Os resultados mostram que todas as dimensões de produtividade são relevantes para explicar as publicações de pares de pesquisadores no Brasil. Especificamente, estar no mesmo estado, pertencer à mesma instituição e à mesma organização possuem associação positiva com o número de publicações. Além disso, interações prévias e contínuas entre os pesquisadores também são considerados fatores importantes.

Palavras-chave:

Conhecimento Científico; Análise de Redes Sociais; Proximidades; Plataforma Lattes

Código JEL:

D85; D83; O34

Abstract:

This study aims to understand the role and influence of the social, institutional, organizational, cognitive and geographical proximities in a collaborative context between researchers in the Brazilian National Innovation System (NIS) in the period between 2009 and 2017. Empirical studies discuss these proximities in countries which have a developed NIS, taken as reference for the present study in the Brazilian NIS, which is considered immature. Although the vast number of studies that handle these dimensions, most of these studies do not consider all of them. To build the network of researchers and extract the proximities, we used the data from the Lattes platform of the National Council for Scientific and Technological Development (CNPq). The results show that all dimensions of productivity are relevant to explain the publications of pairs of researchers in Brazil. Specifically, being in the same state, belonging to the same institution and to the same organization have a positive association with the number of publications. In addition, previous and continuous interactions between researchers are also considered to be important factors.

Keywords:

Scientific Knowledge; Social Network Analysis; Proximity; Lattes platform

Área Temática:

4.3 Sistemas de inovação - nacional, regional, setorial, tecnológico 


\section{Introdução}

As universidades em um Sistema Nacional de Inovação (SNI) imaturo assumem um papel de polos de interação especializados, o que mantém a fronteira do conhecimento e o desenvolvimento tecnológico dos setores mais importantes alinhados a países desenvolvidos, evitando o risco para o país de um maior atraso do SNI (Bernardes \& Albuquerque, 2003; Rapini et al, 2006). A cooperação entre cientistas pesquisadores impulsiona a absorção de conhecimento e eleva o grau de importância das universidades como uma "antena", com ligações internacionais capazes de absorver novas conhecimentos (Cohen \& Levinthal, 1990)

A produção das universidades é movida por pesquisadores cientistas que cujo interesse é focado em publicações revistas de alto impacto, pautadas por um sistema de revisão por pares (Geisler \& Rubenstein, 1989). A produção destes visa o avanço na fronteira do conhecimento que, cada vez mais, exige mais complexidade nas ideias, requerendo especialidades em diversas áreas. Por consequência, colaborações na produção de conhecimento se tornam mais necessárias (Katz \& Martin 1997). As cooperações no meio acadêmico dependem da interação entre esses indivíduos que possuem diferentes características e meios institucionais (Bodas Freitas et al, 2013).

Essa diferença influencia o processo de escolha da parceria na cooperação científica. Características individuais e institucionais podem ser uma fonte da identificação de cooperações (Boschma \& Frenken, 2009). Boschma (2005) traduz essas dimensões em 5 proximidades, a saber, cognitiva, geográfica, organizacional, institucional e social, que serão descritas na revisão da literatura. Nos países emergentes com SNI imaturos a literatura ainda é escassa, limitando-se à interação Universidade-Indústria e a estudos de Análise de Redes Sociais dessas organizações, não englobando todas as proximidades citadas acima.

Este trabalho busca interpretar o papel das proximidades social, cognitiva, geográfica, organizacional e institucional na decisão dos pesquisadores em colaborar, com base no modelo Binomial Negativo Inflado em Zeros (BNIZ). A variável dependente capta a contagem de artigos publicados em comum por pares de autores durante os triênios 2009 a 2011, 2012 a 2014 e 2015 a 2017. As variáveis de proximidades aplicadas empiricamente à cooperação na área científica são recentes na literatura, sendo aplicada somente a países com SNI desenvolvidos (Molina-Morales et al, 2015; Crescenzi et al, 2017; Crescenzi et al, 2016).

Este trabalho é composto por mais 6 seções, além desta introdução. A segunda seção revisa a literatura que abrange a aplicação das proximidades em rede em estudos de difusão de conhecimento. A terceira seção aborda os estudos empíricos acerca das proximidades citadas na seção anterior. Já a quarta seção aborda a estratégia empírica e apresenta estatísticas descritivas da base de dados. Os resultados são apresentados na quinta seção. Na última seção é apresentada a conclusão e as limitações do trabalho.

\section{Proximidade de rede no processo de colaboração científica}

Vários fatores influenciam o pesquisador na tomada de decisão com quem interagir a fim de maximizar a produção científica. O trabalho acadêmico em conjunto pode levar a estudos de maior qualidade, eficiência, compartilhamento da expertise e diminuição de custos. Mesmo os cientistas renomeados estão à procura de coautoria que podem complementar seu domínio acadêmico a fim de fomentar novas ideias, aproveitar habilidades de ambos e compartilhar acesso a diferentes fontes de conhecimento (Smith \& Sotala, 2011). Essa colaboração proporciona melhor entendimento de problemas complexos e que requerem união de várias áreas e pesquisadores.

As colaborações ligam diferentes áreas da academia incorporando nos estudos novas áreas férteis. O preenchimento das lacunas e avanço na fronteira do conhecimento trazido por essa colaboração resulta em artigos científicos inovadores. Trabalhos acadêmicos que possuem coautoria tendem a ser mais citados, especialmente se essas parcerias forem de diferentes países (Smith \& Sotala, 2011). No entanto, mesmo com pares de pesquisadores que se complementam, as parcerias são restritas 
a diversos fatores sociais, físicos e institucionais. Estes são introduzidos em conjunto em cinco dimensões por Boschma (2005). A literatura teórica de proximidade em redes começa a ganhar força na década de 90 (Bellet et al., 1993 apud Balland et al., 2015), com uma variedade de proximidades, como a social, a cognitiva, a institucional, a organizacional e a geográfica. Esta última mais consolidada na literatura (Marshall, 1920; Audretsch \& Feldman, 1996; Anselin et al., 2000; Boschma, 2005; Belussi \& Caldari 2009; Gonçalves et al., 2019).

\subsection{Proximidade Geográfica}

Devido a maior facilidade de transferência de conhecimento, principalmente tácito, pesquisadores geograficamente próximos têm maior propensão a colaborar. A tentativa de identificar o papel da proximidade geográfica vem evoluindo na literatura com diversas metodologias e abordagens. No entanto, deve-se atentar que esse efeito pode ser provocado pelo fato de que relações em rede tendem a ser mediadas geograficamente, o que causaria uma concentração de ligações em rede localizadas que explicaria esse fenômeno espacial. Uma parte do efeito locacional em citação de patentes se deve à autocitação (Criscuolo \& Verspagen, 2008). Essa seria a indicação de difusão de conhecimento na mesma organização ou do mesmo indivíduo.

\subsection{Proximidade Cognitiva}

A difusão do conhecimento muitas vezes está sujeita a conexões com instituições e agentes que têm domínio desse conhecimento. O bom entendimento entre as partes está sujeito à capacidade de absorção, a qual depende da área do conhecimento que esses indivíduos estão inseridos. Se ambos se situam em um mesmo campo tecnológico, esse processo é facilitado. Dado isso, a proximidade cognitiva mensura a distância que os agentes estão nas diversas áreas do conhecimento (Boschma, 2005).

\subsection{Proximidade Social}

Experiências sociais informais nas quais o indivíduo tem ideias são consideradas interações sociais. Conferências, reuniões e encontros são meios de difusão de conhecimento social que, muitas vezes, são difíceis de serem captados em modelos empíricos. A proximidade social é moldada a partir de interações anteriores ou já existentes na rede. São baseadas em confiança e experiência. Logo, os processos tácitos de aprendizagem são facilitados. Relações de amizade, parentesco e interações passadas podem se basear em características em comum dos pesquisadores na rede, sejam elas já predefinidas ou que ocorreram após a interação (Boschma, 2005).

\subsection{Proximidade Institucional}

Instituições são normas, leis, hábitos em comum e incentivos que rege um grupo de indivíduos. Já a proximidade institucional é o inverso da distância que dois indivíduos têm em relação ao contexto institucional. É uma proximidade importante devido à diminuição da incerteza no contexto institucional com normas, leis e cultura similares a nível macro (Boschma, 2005). Apesar de fazer parte de uma rede social, não se deve confundi-la com a distância social, que ocorre ao nível micro (Edquist, 1997). Esta proximidade também leva em conta características locais e regionais. Indivíduos dentro de uma mesma região com características próprias são impactados por esta, além de haver outras estruturas institucionais que operam em conjunto (Balland et al., 2014).

\subsection{Proximidade Organizacional}

A proximidade organizacional refere-se a agentes que se encontram em mesma situação organizacional ou que dividem um mesmo nível hierárquico, seja na mesma ou em diferentes organizações. Esta medida pode ser interpretada como uma variável contínua, onde seus extremos são 
indivíduos completamente autônomos e que possuem ligações fracas, proximidade baixa e indivíduos subordinados a um alto nível hierárquico com ligações fortes e proximidade alta (Boschma, 2005).

Agentes com proximidades organizacional alta exercem um papel relevante na difusão do conhecimento nas periferias da rede (Capaldo et al., 2014), encurtando a distância de atores por terem menor custo de aprendizagem, tendo em vista que tanto a incerteza quanto a possibilidade de oportunismo são diluídos (Boschma, 2005). Esse mecanismo se torna importante na rede de colaboração, contribuindo na formação de gatekeepers $^{1}$ a nível regional e nacional (Lagendijk \& Lorentzen, 2006; Balland et al., 2015).

\section{Estudos empíricos de proximidades}

Trabalhos utilizam as interações prévias a fim de captar a proximidade social, mostrando maior probabilidade de colaborações futuras entre indivíduos que já interagiram (Molina-Morales et al., 2015; Crescenzi et al., 2016; Crescenzi et al., 2017). O estudo de Hong \& Su (2013) também corrobora este resultado ao utilizar as proximidades não-geográficas para inferir a probabilidade de interação universidade-indústria. Os autores concluem que outras proximidades tendem a diminuir o efeito da distância geográfica cujo efeito continua significativo mesmo após este controle.

As proximidades organizacional e institucional podem interferir na transferência de conhecimento e inovação. Nos Estados Unidos da América (EUA), há evidências de que parcerias formadas entre firmas na formação de estratégias de mercado facilitam a transferência de tecnologia (Mowery et al., 1996). Kaygalak \& Reid (2016) analisam a interação universidade-empresa dentro do contexto turco, fazendo-se a diferenciação de tipos organizacionais entre universidade técnica, universidade não-técnica e indústrias. Segundo o teste de independência entre os grupos em relação à medida de proximidade, existe diferença da média entre esses tipos organizacionais. Já na China, a estruturação das universidades que são vinculadas a ministérios específicos e províncias com tipos organizacionais diferentes apontam para uma menor importância da proximidade geográfica quando considerados dois agentes pertencentes ao mesmo ministério (Hong \& Su, 2013). Embora estes trabalhos concordem com expressividade da dimensão organizacional, há indícios de uma menor contribuição desta dimensão, mas ainda significativo, e uma relação de substituição com a dimensão geográfica (Paci et al. 2014; Fernández et al, 2016; Heringa et al., 2016).

A colaboração entre pesquisadores muito próximos cognitivamente se torna prejudicial. É esperado que essa dimensão assuma um formato de U invertido na produtividade inventiva (Cohendet \& Llerena, 1997). Devido a isso os acadêmicos enfrentam um tradeoff entre maior absorção de conhecimento e um grau maior de inovação no ato da colaboração, portanto, uma proximidade muito alta nem sempre reflete maior grau inventivo (Nooteboom, 2000; Nooteboom et al., 2007; Broekel \& Boschma, 2012).

\section{Estratégia Empírica}

O objetivo desse trabalho é verificar o papel dos tipos de proximidades sobre as colaborações científicas. Como base nisso, será utilizado um modelo Binomial Negativo Inflado em Zeros a fim de inferir sobre o número de artigos publicados em colaboração por par de autor.

A contagem do número de publicações em colaboração do par de pesquisadores é a variável dependente. A descrição desta variável por triênio está na Tabela 1. Como é visto na tabela, ela possui uma dispersão maior que a média, o torna a distribuição de Poisson para dados de contagem não adequada, pois não suporta a heterogeneidade não observada dos valores preditos em relação à

\footnotetext{
${ }^{1}$ Gatekeepers são pontes que ligam dois componentes importantes na rede (Borgatti et al, 2013).
} 
sobredispersão (Hilbe, 2011). Portanto, um modelo Binomial Negativo de contagem deve ser utilizado para explicar essas variáveis, já que ele acomoda a heterogeneidade da dispersão.

Há um número grande de pesquisadores na rede e sua maior parte não coopera entre si, caracterizando uma rede esparsa. A fim de inferir sobre a influência da proximidade geográfica, cognitiva, social, organizacional e institucional na rede, será preciso criar observações de ligações de pares fictícias para um contrafactual, as quais se enquadram no grupo de pares de pesquisadores que sempre assumem zero no valor observado. A definição do modelo contrafactual será através da seleção de uma rede aleatória com probabilidade de ligação igualmente distribuída, tal procedimento será descrito posteriormente.

$$
\begin{aligned}
& \quad \text { Publicações do par }_{(i j) t} \\
& =\beta_{0}+\text { Proximidades }_{(i j) t} \beta_{1}+\text { Formação }_{(i j) t} \beta_{2}+\text { Tempo docência }_{i t} \beta_{3} \\
& + \text { Tempo docência }_{j t} \beta_{4}+\text { Betweenness }_{i t} \beta_{5}+\text { Betweenness }_{j t} \beta_{6}+z_{f}+k_{t} \\
& +\varepsilon_{(i j) t}
\end{aligned}
$$

A variável dependente se trata de uma variável de contagem de ligações do par $(i, j)$, sendo, respectivamente, o autor e o coautor. As ligações foram agrupadas nos triênios de 2009 a 2011, 2012 a 2014 e 2015 a 2017. Cada colaboração do par $(i, j)$ no triênio no mesmo artigo indica uma ligação. Publicações do $\operatorname{par}_{(i j) t}$, sendo $i$ o autor, $j$ o coautor, $t$ é a referência do triênio e $z_{f}$ é referente à grande área do conhecimento referente a ambos.

Tabela 1 - Estatísticas de dispersão em torno da média da variável dependente 2009-2017

\begin{tabular}{lll}
\hline Triênio & Média & Desvio-Padrão \\
\cline { 2 - 3 } $2009-2011$ & 1,07461 & 1,4392 \\
$2012-2014$ & 1,27619 & 2,7954 \\
$2015-2017$ & 1,29997 & 2,7241 \\
\hline
\end{tabular}

\subsection{Descrição da base de dados}

Fonte: Elaboração própria a partir de dados da Plataforma Lattes.

A base de dados utilizada é a da Plataforma Lattes, com o período de análise de 2000 a 2017. As estatísticas descritivas se estendem a todo o período, no entanto, por limitação computacional, as regressões incluem somente os 3 últimos triênios, de 2009 a 2017. Essa base se divide nas seguintes partes: publicações de artigos, depósito de patentes, endereço profissional e vínculos de atividades ou trabalho. O número de registros de artigos no período é de 5.822.614 atribuídos a 753.677 autores. Este primeiro valor pode ou não incluir mais de um registro de um mesmo artigo de indivíduos diferentes, ou seja, existem observações de autoria única, autoria solitária, sendo que neste caso só há um registro de um indivíduo, e também de coautoria com mais de um registro de um único título. O número de coautorias é determinado pela quantidade de registros de um mesmo artigo. A mesma lógica é atribuída às patentes. $\mathrm{O}$ número de depósito de patente repetido de diferentes indivíduos indica a cooperação no processo de depósito. O total de registro de depósitos é de 56.924 declarados por 24.114 indivíduos declarados na plataforma

Para construção do filtro que foi aplicado à base descrita foram considerados somente pesquisadores, professores universitários, revisores de periódicos e doutores que publicaram no período de análise. Com isso, a amostra retirada a partir da plataforma lattes conta com 445.390 pesquisadores. Metade destes tem até 5 anos de docência e a média é de 7 anos. A grande maioria, $86 \%$, já teve experiência como docente e $45 \%$ tem doutorado. A região Sudeste é a que concentra maior número de pesquisadores, contando com $35 \%$ da amostra e a região Norte é a menor, com $4 \%$ da amostra. Esse cenário se repete em relação à centralidade betweeness que, em média, no Sudeste é maior que nas outras regiões, seguida por Sul, Nordeste e Centro-Oeste, respectivamente. A Tabela 4 descreve a 
representatividade de cada região na base, a média da centralidade betweeness e a centralidade de grau ponderada média dos pesquisadores dessa região.

Tabela 3 - Descrição das medidas de rede das regiões brasileiras

\begin{tabular}{llll}
\hline Região & Participação da base & $\begin{array}{l}\text { Média centralidade } \\
\text { (Betwenness) }\end{array}$ & $\begin{array}{l}\text { Grau médio } \\
\text { ponderado }\end{array}$ \\
\cline { 2 - 4 } Norte & $5,41 \%$ & $14.412,79$ & 13,04 \\
Nordeste & $20,04 \%$ & $19.749,57$ & 15,78 \\
Sudeste & $45,13 \%$ & $28.317,34$ & 24,05 \\
Sul & $21,32 \%$ & $24.225,10$ & 20,06 \\
Centro-Oeste & $8,11 \%$ & $17.245,38$ & 14,49 \\
\hline
\end{tabular}

Fonte: Elaboração própria a partir dos dados da Plataforma Lattes. Nota: A participação da região na base indica a proporção de pesquisadores da região em relação ao total de pesquisadores, a soma das participações não equivale a totalidade já que há missings. As medidas de rede de centralidade betweeness e grau médio ponderado foram calculados a partir da média dos indivíduos de cada região brasileira que produziram artigos em colaboração.

A Tabela 5 detalha as estatísticas de rede no período 2000 a 2017 das grandes áreas do conhecimento de pesquisas do indivíduo, sendo que o indivíduo pode ser multidisciplinar, ou seja, ter mais de uma grande área. As áreas são classificadas de acordo com a tabela do Conselho Nacional de Desenvolvimento Científico $(\mathrm{CNPq})$. Somente as áreas da saúde, humanas e sociais aplicadas representam mais da metade da base. As áreas com maior colaboração são: ciências biológicas, ciências agrárias e ciências da saúde. A tendência de agrupamento medida pela média do coeficiente de clustering aponta para maior prevalência de grupos na biologia, seguido por ciências agrárias e engenharias.

Tabela 4 - Características das Grandes Áreas do Conhecimento na rede de colaboração

\begin{tabular}{llll}
\hline Grande área do conhecimento & $\begin{array}{l}\text { \% de } \\
\text { indivíduos }\end{array}$ & $\begin{array}{l}\text { Média do coeficiente } \\
\text { de } \text { cluster }\end{array}$ & $\begin{array}{l}\text { Média centralidade } \\
\text { de grau }\end{array}$ \\
\cline { 2 - 4 } Ciências exatas e da terra & $12,08 \%$ & 0,35 & 11,50 \\
Ciências Biológicas & $12,66 \%$ & 0,41 & 20,24 \\
Engenharias & $7,53 \%$ & 0,35 & 8,06 \\
Ciências da Saúde & $21,41 \%$ & 0,35 & 13,36 \\
Ciências Agrárias & $9,12 \%$ & 0,38 & 16,19 \\
Ciências Sociais Aplicadas & $14,54 \%$ & 0,14 & 2,18 \\
Ciências Humanas & $16,52 \%$ & 0,14 & 2,15 \\
Linguísticas, letras e artes & $5,40 \%$ & 0,7 & 1,01 \\
Outros & $0,74 \%$ & 0,33 & 5,15 \\
\hline
\end{tabular}

Fonte: Elaboração própria a partir dos dados da Plataforma Lattes e do software Gephi. Nota: As medidas de rede de coeficiente cluster e centralidade de grau foram calculados a partir da média dos indivíduos de cada grande área do conhecimento brasileira que produziram artigos em colaboração.

O trabalho de Mena-Chalco et al. (2014) utiliza a plataforma Lattes para descrever as medidas de rede de cada área do conhecimento dos pesquisadores. Os autores constatam que as áreas com mais pesquisadores são Ciências da Saúde, Ciências humanas e Ciências Sociais, sendo que as áreas de Ciências Biológicas e Agrárias são as mais colaborativas.

A Tabela 6 descreve a evolução das métricas de rede no tempo. Há um aumento no coeficiente de Cluster que indica maior agrupamento. Isso pode ser consequência de uma rede mais densa e conectada e também da presença de ligações mais fortes indicado pela centralidade de grau.

Os dados mostram que a razão de autores solitários diminui em todos os anos, enquanto que a participação dos autores com parcerias aumenta no tempo. No ano de 2008, a participação dos autores 
únicos representava 24,32\%. Este número, 10 anos depois, cai para 18,96\%. No mesmo período, as participações em coautorias de mais de 3 membros aumentaram em mais de $10 \%$.

\subsection{Variáveis explicativas}

\section{Proximidade social}

Esta variável será construída a partir de interações que o par de indivíduos teve no passado, como utilizado por Crescenzi et al. (2017) e Crescenzi et al. (2016). Este trabalho difere desta literatura, já que considera defasagens temporais de até terceira ordem. Tais interações podem ser em colaboração em artigos ou produção de patente. A construção levou em conta um período de defasagem de interações prévias de até 3 triênios anteriores.

Tabela 6 - Estatísticas descritivas da rede de colaboração dos triênios, Brasil, 2000-2017

\begin{tabular}{|c|c|c|c|c|c|c|}
\hline Triênio & $\begin{array}{l}2000- \\
2002\end{array}$ & $\begin{array}{l}2003- \\
2005\end{array}$ & $\begin{array}{l}2006- \\
2008\end{array}$ & $\begin{array}{l}2009- \\
2011\end{array}$ & $\begin{array}{l}2012- \\
2014\end{array}$ & $\begin{array}{l}2015- \\
2017\end{array}$ \\
\hline Número de autores & 101.537 & 136.688 & 169.93 & 213.932 & 254.193 & 272.264 \\
\hline Grau médio ${ }^{1}$ & 3,12 & 3,86 & 4,77 & 6,08 & 5,18 & 7,77 \\
\hline Grau médio ${ }^{1}$ ponderado & 4,44 & 5,487 & 6,78 & 8,93 & 8,16 & 12,07 \\
\hline $\begin{array}{l}\text { Número médio de artigos sem } \\
\text { colaborações }\end{array}$ & 3,03 & 2,86 & 2,36 & 2,07 & 1,77 & 1,65 \\
\hline \% de nós isolados & 70,22 & 63,35 & 54,06 & 46,07 & 36,15 & 29,26 \\
\hline Coeficiente de cluster médio & 0,60 & 0,62 & 0,645 & 0,658 & 0,631 & 0,657 \\
\hline
\end{tabular}

Fonte: Elaboração própria a partir dados da Plataforma Lattes. Nota: Medidas de rede tomam como base somente os indivíduos que produziram artigos em colaboração

O período de 2000 a 2008 foram 1.440 .760 publicações registradas de 226.236 autores, sendo que $18 \%$ destes foram publicações solo. Após transformar essas observações em pares de autores e agregando todas as publicações é obtido 381.102 vértices de coautoria e 180.209 indivíduos que publicam sozinhos. Os Gráficos 2, 3 e 4 mostram, em porcentagem, o número de cooperações por artigo em 2000 a 2005, 2006 a 2011 e 2012 a 2017, respectivamente. Há uma diferença absoluta entre os três períodos, sendo que o segundo e terceiro períodos tiveram um aumento na publicação de artigos em colaboração. $\mathrm{O}$ aumento de mais de $25 \%^{2}$ dos trabalhos são de autoria única e a maior parte das interações ocorreu somente uma vez (67\%). Interações repetidas representam $27 \%$ da amostra, estes são os potenciais laços mais fortes na rede. A Tabela 7 descreve a variável explicativa de proximidade social de coautorias segregada por triênios e por defasagem temporal. A construção desta foi por três variáveis binárias cujo valor assume 1 quando o par de autores já teve alguma interação prévia na defasagem de referência. Há um crescimento no tempo de interações prévias e isso condiz com a literatura, já que, com o tempo, a fronteira do conhecimento se torna mais complexa e demanda mais cooperações e interações repetidas são mais frequentes visto que há menos incerteza nessa parceria (Danell, 2014).

\footnotetext{
${ }^{2}$ Este é o valor observado, criado a partir de das referências dos artigos publicados, ou seja, o autor acusou que este artigo é de autoria única. Este valor diverge do valor da rede criada, já que para montagem da rede foi necessário a correspondência das coautorias pelo título da publicação, o que nem sempre é exatamente igual em todos, mesmo depois de todas as correções manuais. A Tabela 7 mostra tal divergência, que nos triênios finais, aqueles que serão utilizados na regressão, convergem para o valor observado citado.
} 
É possível observar que as defasagens mais recentes são mais representativas. A frequência de presença de interação na primeira defasagem é mais que o dobro na segunda defasagem em todos os triênios. Essa tendência é lógica, visto que projetos desenvolvidos por pesquisadores podem produzir vários artigos os quais são aceitos e publicados subsequentemente. As interações prévias de primeira ordem estão correlacionadas com a de segunda ordem. Das 373.901 interações em $t-1,107.481$ dessas se repetem em $t$-2, e 34.752 pares de indivíduos mantiveram relação nos 3 períodos de defasagem. Devido a isso, é preciso atentar para esta variável ao incluí-la no modelo, pois há risco de colineariadade. Esse raciocínio não é empregado no copatenteamento ${ }^{3}$, já que são poucas observações. Para esse caso, foi criado somente uma variável binária que acusa se o par de autores já patenteou em conjunto anteriormente.

\section{Proximidade institucional}

A variável de proximidade institucional será construída a partir de dados informados pelos pesquisadores do currículo Lattes. Estes informam à qual instituição estiveram vinculados durante sua vida acadêmica. Para isso, foram criadas Dummies que remetem às instituições mais frequentes na amostra, como institutos de pesquisa aplicados, Empresa Brasileira de Pesquisa Agropecuária (EMBRAPA) e universidades públicas. Outra Dummy criada foi a de correspondência exata de instituição, que recebe valor igual a um quando o par já teve vínculo na mesma instituição.

\section{Proximidade organizacional}

Indivíduos que possuem passagem por empresas ou universidade vivenciam tipos organizacionais diferentes, como normas, regras e incentivos que divergem. As variáveis criadas permitem entender se a relação intra e inter organizacionais são mais ou menos cooperativas. Dummies dos pares foram criadas a fim de discriminar se o par de indivíduos tem experiência universitária ou no setor produtivo, também se ambos tiveram carreira em universidade e em empresa. Essa análise de pertencimento ao setor produtivo ou à universidade e se estes grupos diferem no posicionamento da rede já fora feita por Kaygalak \& Reid (2016).

Tabela 7 - Descrição e distribuição da variável social de ligações prévias no tempo 2009-2017

\begin{tabular}{llll}
\hline Triênio & $\mathrm{t}-1$ & $\mathrm{t}-2$ & $\mathrm{t}-3$ \\
\cline { 2 - 4 } $2015-2017$ & 183.645 & 78.502 & 10.912 \\
$2012-2014$ & 123.908 & 50.342 & 20.869 \\
$2009-2011$ & 66.348 & 25.775 & 33.763 \\
Média (Desvio-Padrão) & $0,017(0,130)$ & $0,007(0,084)$ & $0,003(0,055)$ \\
\hline
\end{tabular}

Fonte: Elaboração própria a partir de dados da Plataforma Lattes

\section{Proximidade Cognitiva}

A variável cognitiva indica à qual área do conhecimento ambos os pesquisadores pertencem. Como visto na Tabela 5, há uma diferença grande entre os grupos que cooperam de acordo com sua grande área do conhecimento. A construção dessa variável leva a inclusão de várias dummies por grande área que assumem 1 quando ambos os indivíduos do par correspondem àquela grande área, caso contrário 0. No total são 9 grandes áreas do conhecimento definidas pela tabela do CNPq: Ciências exatas e da terra; Ciências Biológicas; Engenharias; Ciências da Saúde; Ciências Agrárias; Ciências Sociais Aplicadas; Ciências Humanas; Linguísticas, letras e artes; e Outros.

\footnotetext{
${ }^{3}$ Foram observadas 19.656 interações prévias em copatenteamento na rede com média de 0,001 e 0,030 de desviopadrão
} 


\section{Proximidade Geográfica}

Como a base de dados se limitava a um número de missings de cidades muito grande, optou-se por considerar a Unidade da Federação para construção da variável de proximidade geográfica. Esta é uma variável binária que, caso ambos os autores do par sejam do mesmo estado informado, assume o valor de 1 , caso contrário 0.

\section{Variáveis de controle}

De acordo com o vínculo institucional que os pesquisadores apresentaram na plataforma, foi possível identificar quais desses se enquadraram em atividades de docência. A partir desse indicador foram somados os meses que algum tipo de docência ocorreu. Essa variável determina o total de meses em logaritmo tanto do pesquisador $i$ quanto do $j$. Seguindo o mesmo raciocínio, onde são consideradas duas variáveis para o par de pesquisadores, acrescenta-se as medidas de betweeness do par. A terceira variável de controle é binária, a qual indica se ambos do par são doutores.

Apresentadas as variáveis do modelo, a Tabela 10 apresenta as correlações entre estas. As variáveis que são mais correlacionadas com a dependente são as de interações prévias, a dummy do mesmo estado e o logaritmo dos betweeneess de $i$ e $j$. As variáveis independentes não apresentam correlação muito alta para ao ponto de manifestar multicolinearidade na regressão.

\subsection{Construção do contrafactual}

No período analisado de 2009 a 2017, foram mapeados 445.390 indivíduos que, na rede, são nós que têm potencial de ligação. Caso todos estes nós se ligassem, teríamos uma rede com 99.185.903.355 possíveis laços. Tal situação hipotética é irreal, visto que a coautoria de um único indivíduo com outros 445.389 autores é impossível. 
Gráfico 2 - Distribuição de número de coautorias por artigo 2000-2002

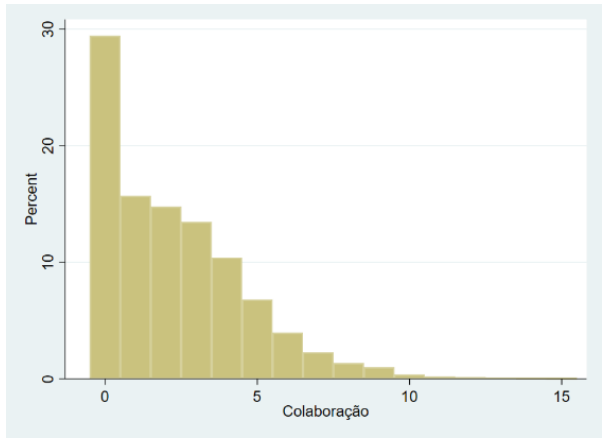

Fonte: Elaboração própria a partir de dados da plataforma lattes. Nota: A primeira coluna referente ao 0 , indica o número de artigo publicados em autoria única

\section{Gráfico 4 - Distribuição de número de coautorias por artigo 2006-2008}

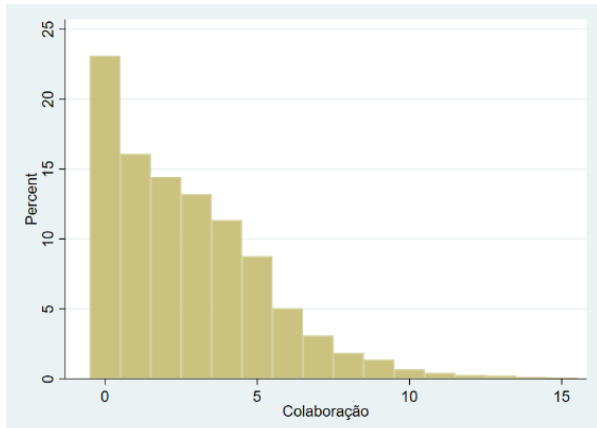

Fonte: Elaboração própria a partir de dados da plataforma lattes. Nota: A primeira coluna referente ao 0 , indica o número de artigo publicados em autoria única

\section{Gráfico 6 - Distribuição de número de coautorias por artigo 2012-2014}

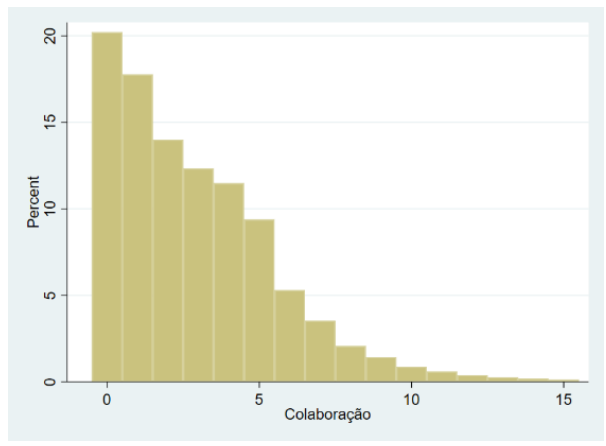

Fonte: Elaboração própria a partir de dados da plataforma lattes. Nota: A primeira coluna referente ao 0 , indica o número de artigo publicados em autoria única
Gráfico 3 - Distribuição de número de coautorias por artigo 2003-2005

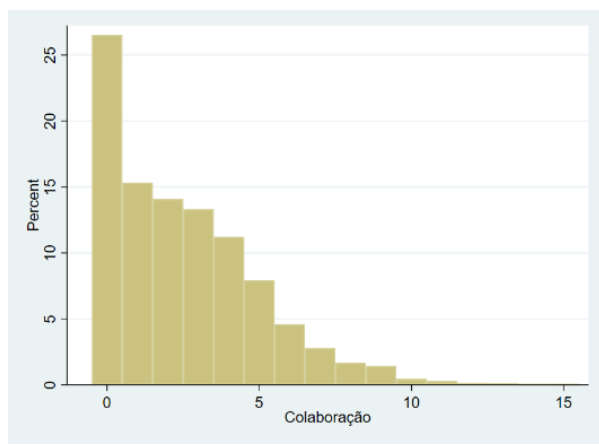

Fonte: Elaboração própria a partir de dados da plataforma lattes. Nota: A primeira coluna referente ao 0 , indica o número de artigo publicados em autoria única

\section{Gráfico 5 - Distribuição de número de coautorias por artigo 2009-2012}

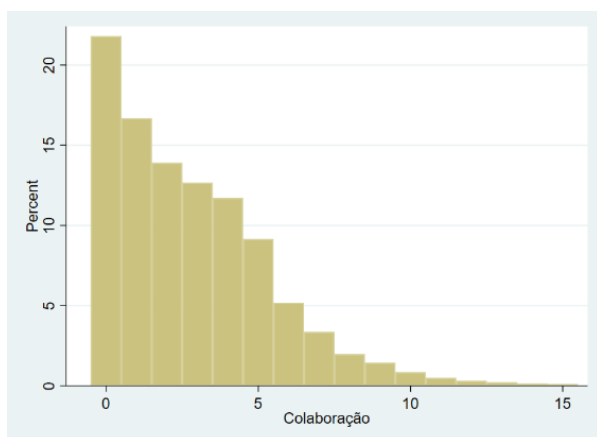

Fonte: Elaboração própria a partir de dados da plataforma lattes. Nota: A primeira coluna referente ao 0 , indica o número de artigo publicados em autoria única

\section{Gráfico 7 - Distribuição de número de coautorias por artigo 2015-2017}

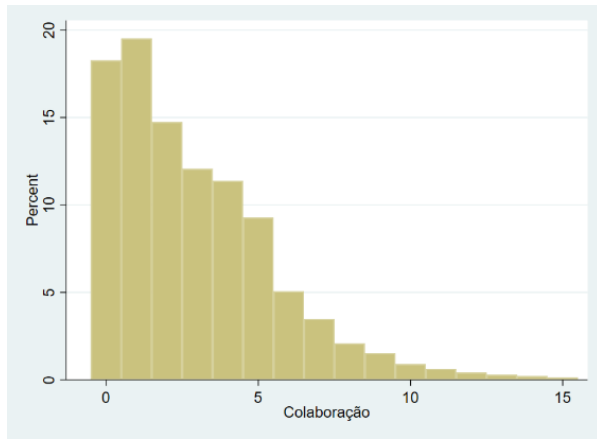

Fonte: Elaboração própria a partir de dados da plataforma lattes. Nota: A primeira coluna referente ao 0 , indica o número de artigo publicados em autoria única 
Dada tal situação, são criadas redes fictícias nas quais as ligações podem ser aleatórias ou definidas por características similares dos indivíduos (Chandrasekhar, 2015). Para comparação da base de dados onde há ligações entre os indivíduos, é preciso criar um contrafactual onde $g_{i j}=0$. Em parte, tais modelos criam uma rede aleatória onde há uma probabilidade de ligação distribuída entre os indivíduos.

Neste trabalho será adotado o método para criação do contrafactual de Ërdos-Renyi (ERM) (Erdös e Renyi, 1959), descrito por Chandrasekhar (2015). O modelo construído de forma aleatória conta com todos os pesquisadores filtrados, considerando os que publicam em solo e em coautoria.

A probabilidade de formação de ligação dos nós na rede é de 0,05 . A Tabela 8 mostra a divisão das observações entre o contrafactual, onde $g_{i j}=0$ e as ligações verdadeiras, $g_{i j} \geq 1$. A Tabela 10 traz valores da base por completa. Sendo assim, as estatísticas levam em conta o contrafactual, que é a maior parte das observações, e os pares de pesquisadores observados.

Tabela 8 - Divisão do contrafactual nos triênios, 2009-2017

\begin{tabular}{llll}
\hline $\mathrm{Y}$ & $2009-2011$ & $2012-2014$ & $2015-2017$ \\
\hline Laço não formado $\left(g_{i j}=0\right)$ & $94,49 \%$ & $91,19 \%$ & $89,44 \%$ \\
Laço formado $\left(g_{i j} \geq 1\right)$ & $5,51 \%$ & $8,81 \%$ & $10,56 \%$ \\
Total de laços & 7.070 .810 & 7.326 .663 & 7.469 .855 \\
\hline
\end{tabular}

Fonte: Elaboração própria a partir de dados da Plataforma Lattes

Tabela 9 - Estatísticas descritivas das variáveis de interesse do total de observações, 2009-2017

\begin{tabular}{lllll}
\hline Variável & Média & Desvio-Padrão & Mínimo & Máximo \\
\hline ContArtigo & 0,134 & 0,880 & 0 & 603 \\
IntPrev1 & 0,017 & 0,130 & 0 & 1 \\
IntPrev2 & 0,007 & 0,084 & 0 & 1 \\
IntPrev3 & 0,003 & 0,055 & 0 & 1 \\
IntPat & 0,001 & 0,074 & 0 & 1 \\
MesmaUF & 0,134 & 0,341 & 0 & 1 \\
MesmaTipoInst & 0,306 & 0,461 & 0 & 1 \\
MesmaInst & 0,011 & 0,221 & 0 & 1 \\
MesmaUni & 0,946 & 0,226 & 0 & 1 \\
MesmaEmp & 0,314 & 0,226 & 0 & 1 \\
MesmaUnivPubl & 0,449 & 0,497 & 0 & 1 \\
MsmGrandArea & 0,052 & 0,222 & 0 & 1 \\
d & 4,613 & 4,766 & $-6,557$ & 17,303 \\
LnBetweenessj & 3,707 & 4,707 & $-6,497$ & 17,303 \\
LnTempDocen $i$ & 3,324 & 1,887 & 0 & 6,825 \\
LnTempDocenj $j$ & 3,672 & 1,746 & 0 & 6,825 \\
AmbosDoutor & 0,237 & 0,425 & 0 & 1 \\
\hline
\end{tabular}

Fonte: Elaborado a partir de dados da plataforma lattes.

\section{Resultados}

Os resultados da regressão BNIZ em painel do período de 2009-2011 são descritos na Tabela 11. Os resultados foram divididos de acordo com o acréscimo de uma defasagem a cada modelo até que foram incluídas todas as variáveis de proximidade social. Devido a dificuldades computacionais, já que se tratam de mais de 20 milhões de observações e 27 parâmetros, foi feita uma amostra de $10 \%$ destes, totalizando 2.182.666 observações. Essa amostra deve ser representativa da base, pois já é um grande 
número de observações e, segundo a Lei dos Grandes Números, isso garante a consistência dos coeficientes da amostra.

Para melhor visualização as variáveis foram agrupadas em partes: proximidade social, proximidade geográfica, proximidade institucional, proximidade organizacional, e variáveis de controle. As variáveis da proximidade cognitiva foram omitidas para melhor visualização.

A primeira variável, referente à proximidade social, é acrescida a cada regressão com defasagem temporal até $t$-3. Em todos os modelos as variáveis são significativas. Assim como os outros parâmetros, os coeficientes de defasagem sofrem pouca alteração nos modelos. A regressão indica que as interações prévias até dois triênios anteriores influenciam positivamente a cooperação entre o mesmo par de indivíduos, embora o coeficiente diminua em $t$-2. A defasagem de 3 triênios prévios apresenta sinal negativo e significativo, mas um valor absoluto pequeno. Isto é, ligações prévias de triênios imediatos à observação são mais propícias à colaboração em contraste às ligações mais antigas. Vale ressaltar que essa variável é composta por maioria de ligações que se repetem em $t-2$ e $t$-1. Esse resultado condiz com a literatura, que mostra uma relação benéfica entre a proximidade social e a cooperação (Heringa $e t$ al, 2014; Heringa et al, 2016; Molina-Morales, 2015; Crescenzi et al, 2016; Fernández et al, 2016; Crescenzi et al, 2017). Em acréscimo a essa análise, uma sétima coluna foi inserida a fim de distinguir somente interações prévias contínuas, aquelas que se mantiveram durante os 3 triênios. O sinal positivo e significativo sugere um efeito similar às anteriores, no entanto sua magnitude foi menor. Mesmo assim, o resultado atesta que pode haver confiança entre os pares que cooperam continuamente, com efeitos benéficos sobre a produção científica.

O coeficiente da variável de proximidade geográfica foi positivo e significativo. Isso mostra que indivíduos na mesma Unidade da Federação tendem a ser mais colaborativos. Tal resultado é condizente com a literatura (Heringa et al, 2014; Molina-Morales et al, 2015; Crescenzi et al. 2016). Já a terceira parte é referente à proximidade institucional, composta por duas variáveis binárias. A primeira indica quando o par de indivíduo está em instituições de pesquisa públicas e o segundo quando ambos estão na mesma instituição. Estas variáveis foram positivas e significativas. Vale ressaltar que o coeficiente da segunda variável possui uma magnitude maior. A literatura diverge a respeito desses resultados, sendo que estes resultados estão de acordo com os estudos de Crescenzi et al. (2017) e Broekel \& Mueller (2018), enquanto Molina-Morales et al (2015) encontram um resultado negativo e pouco significativo após controlar por todas as proximidades e sugere que o papel da dimensão institucional reforça o da organizacional, podendo atuar como complementos. 
Tabela 10 - Correlação entre as variáveis do modelo

\begin{tabular}{|c|c|c|c|c|c|c|c|c|c|c|c|c|c|c|c|c|c|}
\hline & $(1)$ & (2) & (3) & (4) & $(5)$ & (6) & (7) & (8) & (9) & (10) & (11) & (12) & (13) & (14) & (15) & (16) & (17) \\
\hline $\begin{array}{l}\text { (1) Contagem } \\
\text { (dependente) }\end{array}$ & 1.00 & & & & & & & & & & & & & & & & \\
\hline (2) IntPrev1 & 0.38 & 1.00 & & & & & & & & & & & & & & & \\
\hline (3) IntPrev2 & 0.27 & 0.44 & 1.00 & & & & & & & & & & & & & & \\
\hline (4) IntPrev3 & 0.19 & 0.29 & 0.43 & 1.00 & & & & & & & & & & & & & \\
\hline (5) MsmTipInst & 0.03 & 0.03 & 0.03 & 0.02 & 1.00 & & & & & & & & & & & & \\
\hline (6) MsmUni & 0.02 & 0.02 & 0.02 & 0.01 & 0.01 & 1.00 & & & & & & & & & & & \\
\hline (7) MsmEmp & 0.08 & 0.08 & 0.06 & 0.04 & 0.15 & 0.05 & 1.00 & & & & & & & & & & \\
\hline (8) MsmUF & 0.21 & 0.19 & 0.13 & 0.09 & 0.03 & 0.03 & 0.06 & 1.00 & & & & & & & & & \\
\hline (9) MsmUniPub & 0.07 & 0.06 & 0.04 & 0.02 & -0.02 & 0.22 & 0.03 & 0.03 & 1.00 & & & & & & & & \\
\hline (10) MsmMest & -0.04 & -0.04 & -0.03 & -0.02 & -0.03 & 0.01 & -0.05 & -0.04 & -0.04 & 1.00 & & & & & & & \\
\hline (11) AmbosDout & 0.17 & 0.18 & 0.13 & 0.09 & 0.06 & 0.09 & 0.13 & 0.15 & 0.16 & -0.20 & 1.00 & & & & & & \\
\hline (12) LigaPat & 0.08 & 0.07 & 0.06 & 0.05 & 0.01 & 0.00 & 0.02 & 0.04 & 0.01 & -0.01 & 0.03 & 1.00 & & & & & \\
\hline (13) MsmInst & 0.10 & 0.11 & 0.07 & 0.05 & 0.00 & 0.01 & 0.00 & 0.10 & 0.04 & -0.01 & 0.03 & 0.02 & 1.00 & & & & \\
\hline (14) LnBetween & 0.14 & 0.14 & 0.11 & 0.08 & 0.04 & 0.02 & 0.08 & 0.10 & 0.05 & -0.05 & 0.14 & 0.03 & 0.02 & 1.00 & & & \\
\hline (15) LnCobetwee & 0.13 & 0.13 & 0.10 & 0.07 & 0.03 & 0.02 & 0.06 & 0.09 & 0.04 & -0.04 & 0.12 & 0.02 & 0.01 & 0.07 & 1.00 & & \\
\hline (16) LnTempdoce & 0.04 & 0.05 & 0.05 & 0.05 & 0.02 & 0.06 & 0.04 & 0.05 & 0.01 & -0.08 & 0.19 & 0.01 & 0.00 & 0.15 & 0.01 & 1.00 & \\
\hline (17) LnCotempdo & 0.03 & 0.04 & 0.04 & 0.04 & 0.01 & 0.05 & 0.01 & 0.04 & 0.00 & -0.08 & 0.19 & 0.01 & 0.00 & 0.01 & 0.10 & 0.01 & 1.00 \\
\hline
\end{tabular}

Fonte: Elaboração própria a partir dos dados da plataforma Lattes 
As variáveis da quarta parte descrevem a proximidade organizacional. Essas variáveis binárias foram criadas a fim de captar a relação de pares que estão sob o mesmo tipo organizacional. A primeira dummy, que indica se o par já teve algum vínculo com universidade, mostra-se positiva e significava, assim como a dummy que indica se ambos declararam vínculo com empresas na plataforma. Estes resultados indicam que a proximidade organizacional ajuda na colaboração entre os agentes da rede, mesmo que em longas distâncias. O estudo de Hong \& Su (2012) confirma esses resultados e fortalece o argumento de que a proximidade organizacional atua como um fator que "encurta" as distâncias geográficas entre pesquisadores.

A quinta parte mostra o coeficiente de variáveis binárias que indicam interação entre pesquisadores da mesma grande área do conhecimento. Como discutido na revisão de literatura, cada área do conhecimento tem sua particularidade e apresenta número de produções científicas em proporções diferentes. Por isso, é um fator importante de controle, pois mede a proximidade cognitiva entre os agentes. Os resultados mostram que todas as grandes áreas tiveram um resultado significativo e na direção de aumentar o número de ligações do par. As áreas que merecem destaque por terem coeficientes em valor absoluto maior são as Ciências Agrárias, as Ciências Exatas e da Terra, as Ciências Biológicas e Ciências da Saúde. Esses achados são condizentes com os números apresentados por MenaChalco et al (2014) que mostram maior produção e cooperação desses campos científicos.

As variáveis de controle do modelo são apresentadas na sexta parte. Optou-se por incluir as características dos dois pesquisadores para as variáveis de betweeness e tempo de docência. A centralidade de entrelaçamento apresentou um sinal positivo e significativo já esperado pela literatura, mas seu coeficiente foi pequeno em valor absoluto, pois os valores são contínuos e possuem uma média absoluta bem maior que as outras variáveis. Esse raciocínio também se emprega em relação ao tempo de docência, uma variável contínua. Entretanto, seu coeficiente é negativo e significativo. Isso é plausível na literatura, já que estudos mostram que a produtividade dos pesquisadores diminui com a idade (Bonaccorsi \& Daraio, 2003). 
Tabela 11 - Determinantes do número de publicações por pares de pesquisadores no Brasil (2009-2017). Estimação por Modelo Binomial Negativo Inflado.

\begin{tabular}{|c|c|c|c|c|c|c|c|}
\hline Variáveis & 1 & 2 & 3 & 4 & 5 & 6 & 7 \\
\hline \multicolumn{8}{|l|}{ Proximidade social } \\
\hline Interação Prévia t-1 & - & - & - & $\begin{array}{l}1,16^{* * * *} \\
(0,01)\end{array}$ & $\begin{array}{l}1,11 * * * \\
(0,01)\end{array}$ & $\begin{array}{l}1,11 \text { *** } \\
(0,01)\end{array}$ & - \\
\hline Interação Prévia t-2 & - & - & - & - & $\begin{array}{l}0,19 * * * \\
(0,01)\end{array}$ & $\begin{array}{l}0,20 * * * \\
(0,01)\end{array}$ & - \\
\hline Interação Prévia t-3 & - & - & - & - & - & $\begin{array}{l}-0,04 * * \\
(0,01)\end{array}$ & - \\
\hline Interação Prévia Patente & - & - & $\begin{array}{l}0,30 * * * \\
(0,01)\end{array}$ & $\begin{array}{l}0,16 * * * \\
(0,01)\end{array}$ & $\begin{array}{l}0,16 * * * \\
(0,01)\end{array}$ & $\begin{array}{l}0,16 * * * \\
(0,01)\end{array}$ & - \\
\hline Interação Contínua & - & - & - & - & - & - & $\begin{array}{l}0,86 * * * \\
(0,02)\end{array}$ \\
\hline \multicolumn{8}{|l|}{ Proximidade Geográfica } \\
\hline Dummy UF & $\begin{array}{l}1,05 * * * \\
(0,01)\end{array}$ & $\begin{array}{l}1,05 * * * \\
(0,01)\end{array}$ & $\begin{array}{l}1,00 * * * \\
(0,01)\end{array}$ & $\begin{array}{l}0,90 * * * \\
(0,01)\end{array}$ & $\begin{array}{l}0,90 * * * \\
(0,01)\end{array}$ & $\begin{array}{l}0,90 * * * \\
(0,01)\end{array}$ & $\begin{array}{l}0,96 * * * \\
(0,01)\end{array}$ \\
\hline \multicolumn{8}{|l|}{ Proximidade institucional } \\
\hline Dummy Tipo Institucional & - & - & $\begin{array}{l}0,02 * * * \\
(0,01)\end{array}$ & $\begin{array}{l}0,01 * * \\
(0,01)\end{array}$ & $\begin{array}{l}0,01 \\
(0,01)\end{array}$ & $\begin{array}{l}0,01 \\
(0,01)\end{array}$ & $\begin{array}{l}0,02 * * * \\
(0,01)\end{array}$ \\
\hline Dummy Instituição & - & - & $\begin{array}{l}0,54 * * * \\
(0,01)\end{array}$ & $\begin{array}{l}0,41 * * * \\
(0,01)\end{array}$ & $\begin{array}{l}0,41 * * * \\
(0,01)\end{array}$ & $\begin{array}{l}0,41 \text { *** } \\
(0,01)\end{array}$ & $\begin{array}{l}1,26 * * * \\
(0,01)\end{array}$ \\
\hline
\end{tabular}

Nota: Erros-padrão entre parênteses. Nível de significância: * 5\%, **1\%,***0,1\%. Variável Dependente: Contagem do número de artigos publicados pelo par de pesquisadores. As áreas do conhecimento inclusas são Ciências exatas e da terra; Ciências Biológicas; Engenharias; Ciências da Saúde; Ciências Agrárias; Ciências Sociais Aplicadas; Ciências Humanas; Linguísticas, letras e artes; e Outros. 
Tabela 11 - Determinantes do número de publicações por pares de pesquisadores no Brasil (2009-2017). Estimação por Modelo Binomial Negativo Inflado.

\begin{tabular}{|c|c|c|c|c|c|c|c|}
\hline Variáveis & 1 & 2 & 3 & 4 & 5 & 6 & 7 \\
\hline \multicolumn{8}{|l|}{ Proximidade organizacional } \\
\hline Dummy Universidade & - & $\begin{array}{l}0,26^{* *} \\
(0,01)\end{array}$ & $\begin{array}{l}0,05^{* *} \\
(0,02)\end{array}$ & $\begin{array}{l}0,07 * * * \\
(0,02)\end{array}$ & $\begin{array}{l}0,08^{* * * *} \\
(0,02)\end{array}$ & $\begin{array}{l}0,08 * * * \\
(0,02)\end{array}$ & $\begin{array}{l}0,06^{* *} \\
(0,02)\end{array}$ \\
\hline \multirow[t]{2}{*}{ Dummy Empresa } & - & $0,09 * * *$ & $0,10 * * *$ & $0,08 * * *$ & $0,08 * * *$ & $0,08^{* * *}$ & $0,10^{* * *}$ \\
\hline & & $(0,01)$ & $(0,01)$ & $(0,01)$ & $(0,01)$ & $(0,01)$ & $(0,01)$ \\
\hline Dummy Universidade Pública & - & $\begin{array}{l}0,26 * * * \\
(001)\end{array}$ & $0,23 * * *$ & $0,23 * * *$ & $0,24 * * *$ & $0,24 * * *$ & $0,22 * * *$ \\
\hline \multicolumn{8}{|l|}{ Controle } \\
\hline $\operatorname{Ln}($ Betweeness i) & $\begin{array}{l}0,20 * * * \\
(0,00)\end{array}$ & $\begin{array}{l}0,20 * * * \\
(0,00)\end{array}$ & $\begin{array}{l}0,20 * * * \\
(0,00)\end{array}$ & $\begin{array}{l}0,19 * * * \\
(0,00)\end{array}$ & $\begin{array}{l}0,18^{* * * *} \\
(0,00)\end{array}$ & $\begin{array}{l}0,18 * * * \\
(0,00)\end{array}$ & $\begin{array}{l}0,20 * * * \\
(0,00)\end{array}$ \\
\hline $\operatorname{Ln}($ Betweeness $j)$ & $\begin{array}{l}0,23 * * * \\
(0,00)\end{array}$ & $\begin{array}{l}0,23 * * * \\
(0,00)\end{array}$ & $\begin{array}{l}0,23 * * * \\
(0,00)\end{array}$ & $\begin{array}{l}0,21 * * * \\
(0,00)\end{array}$ & $\begin{array}{l}0,21 * * * \\
(0,00)\end{array}$ & $\begin{array}{l}0,21 * * * \\
(0,00)\end{array}$ & $\begin{array}{l}0,23 * * * \\
(0,00)\end{array}$ \\
\hline Ln(Tempo Docência $i$ ) & $\begin{array}{l}-0,04 * * * \\
(0,00)\end{array}$ & $\begin{array}{l}-0,05 * * * \\
(0,00)\end{array}$ & $\begin{array}{l}-0,05^{* * * *} \\
(0,00)\end{array}$ & $\begin{array}{l}-0,05 * * * \\
(0,00)\end{array}$ & $\begin{array}{l}-0,06 * * * \\
(0,00)\end{array}$ & $\begin{array}{l}-0,06 * * * \\
(0,00)\end{array}$ & $\begin{array}{l}-0,05^{* * * *} \\
(0,00)\end{array}$ \\
\hline Ln(Tempo Docência $j$ ) & $\begin{array}{l}-0,09 \text { *** } \\
(0,00)\end{array}$ & $\begin{array}{l}-0,09 * * * \\
(0,00)\end{array}$ & $\begin{array}{l}-0,09 * * * \\
(0,00)\end{array}$ & $\begin{array}{l}-0,10^{* * *} \\
(0,00)\end{array}$ & $\begin{array}{l}-0,10 \text { *** } \\
(0,00)\end{array}$ & $\begin{array}{l}-0,10 * * * \\
(0,00)\end{array}$ & $\begin{array}{l}-0,09 \text { **** } \\
(0,00)\end{array}$ \\
\hline Ambos Doutores & $\begin{array}{l}0,19 * * * \\
(0,01)\end{array}$ & $\begin{array}{l}0,18 * * * \\
(0,01)\end{array}$ & $\begin{array}{l}0,18 * * * \\
(0,01)\end{array}$ & $\begin{array}{l}0,08^{* * * *} \\
(0,01)\end{array}$ & $\begin{array}{l}0,08 * * * \\
(0,01)\end{array}$ & $\begin{array}{l}0,08 * * * \\
(0,01)\end{array}$ & $\begin{array}{l}0,17 * * * \\
(0,01)\end{array}$ \\
\hline Constante & $\begin{array}{l}5,40 * * * \\
(0,01)\end{array}$ & $\begin{array}{l}-5,54 * * * \\
(0,02)\end{array}$ & $\begin{array}{l}-5,56^{* * * *} \\
(0,02)\end{array}$ & $\begin{array}{l}-5,34 * * * \\
(0,02)\end{array}$ & $\begin{array}{l}-5,33 * * * \\
(0,02)\end{array}$ & $\begin{array}{l}-5,33 * * * \\
(0,02)\end{array}$ & $\begin{array}{l}-5,52 \text { *** } \\
(0,02)\end{array}$ \\
\hline Dummies de áreas do & Sim & Sim & Sim & Sim & Sim & Sim & Sim \\
\hline
\end{tabular}
Nota: Erros-padrão entre parênteses. Nível de significância: * 5\%,**1\%, ***0,1\%. Variável Dependente: Contagem do número de artigos publicados pelo
par de pesquisadores. ${ }^{1}$ As áreas do conhecimento inclusas são Ciências exatas e da terra; Ciências Biológicas; Engenharias; Ciências da Saúde; Ciências Agrárias; Ciências Sociais Aplicadas; Ciências Humanas; Linguísticas, letras e artes; e Outros. 


\section{Conclusões}

Este trabalho focou nos tipos de proximidades e nos seus impactos sobre a colaboração científica. Em particular, o artigo explorou essas dimensões ao nível de indivíduos em um país com um SNI em construção. Neste contexto, a inserção das cinco proximidades de rede preenche a lacuna nacional e, em relação à literatura internacional, fornece evidências de países em desenvolvimento. Os resultados expressam a importância das dimensões de proximidade dos pesquisadores na decisão de colaborar.

As diferenças dos números de colaborações entre grupos da mesma Grande Área do Conhecimento são fundamentais para o entendimento e controle das outras proximidades. Grupos de pesquisadores das áreas de Ciências Biológicas, Ciências da Saúde e Ciências Agrárias são as mais colaborativas. $\mathrm{O}$ pertencimento do par de pesquisadores à mesma área do conhecimento facilita a colaboração, implicando na expressividade da proximidade cognitiva.

As proximidades organizacional e institucional também impactam positivamente o processo de colaboração, embora esta primeira não tenha um grande impacto em relação às outras dimensões. $\mathrm{O}$ maior coeficiente encontrado é o da variável dummy que mede o pertencimento à mesma instituição. No entanto, pertencer à mesma organização, como empresas, universidades e universidades públicas, também mostra associação positiva com a produção científica dos pares. Estes achados mostram o papel importante da instituição a nível macro e micro, e podem indicar diluição da incerteza e oportunismo na formação de equipes de pesquisa.

As relações prévias mantidas pelos pesquisadores durante o triênio, que são representadas pela proximidade social, sugerem que essas interações proporcionam novas oportunidades de colaboração. O efeito é também positivo nos casos de pares de pesquisadores que já interagiram continuamente nos últimos dois triênios. Isso mostra que o processo de conhecimento mútuo entre os pares encoraja a produção científica exceto se os pares possuíram interações longínquas, ou seja, em mais de 6 anos no tempo (interações em t-3). Por outro lado, interações contínuas dos mesmos pares de pesquisadores afetam positivamente a produção científica, o que também pode significar confiança mútua entre os pares de pesquisadores. A proximidade geográfica interfere positivamente na decisão de colaboração dos acadêmicos, ainda que seja medida neste estudo como pertencimento ao mesmo estado. Outros resultados como grau de centralidade dos pesquisadores na rede e tempo de docência possuem resultados que mostram associação positiva e negativa, respectivamente. Num caso, revela-se que a posição do pesquisador na rede afeta positivamente a colaboração dos pares. No outro, a produtividade decrescente com o tempo de docência revela a natureza do ciclo de vida do pesquisador, o qual com o do tempo se envolve com outras funções além da publicação de artigos científicos em si.

Este estudo tem algumas limitações que valem observar. Primeiro, leva em conta as colaborações que se formaram, ou seja, os insucessos nas produções em colaboração são desconsiderados. Segundo, somente interações acadêmicas que levam ao produto artigo são consideradas, sejam elas formais ou informais. Terceiro, as variáveis de proximidade são todas representadas por dummies; e a unidade de referência geográfica é a Unidade da Federação, o que deve implicar em menos precisão da dimensão geográfica, visto que os estados brasileiros têm grande dimensões.

\section{Referências}

ALBUQUERQUE, E. M. Notas sobre os determinantes tecnológicos do processo de catching-up: uma introdução à discussão sobre o papel dos sistemas nacionais de inovação na periferia. Estudos Econômicos, São Paulo, v.27(2), 1997. 
ALBUQUERQUE, E. M.; SILVA, L. A.; PÓVOA, L. Diferenciação Intersetorial na interação entre empresas e universidades no Brasil. São Paulo em Perspectiva, v. 19(1), p. 95-104, 2005.

ALBUQUERQUE, E.; SUZIGAN, M.; KRUSS, G.; LEE, K. Developing National Systems of Innovation, Edward Elgar, 2015.

ALBA, R.; KADUSHIN, C. The Intersection of Social Circles: A New Measure of Social Proximity in Networks Sociological Methods \& Research v. 5(1), 1976.

ANSELIN, L.; VARGA, A.; ACS, Z. Geographic and sectoral characteristics of academic knowledge externalities. Papers in Regional Science v. 79, p. 435-445, 2000.

ANSELIN, L.; VARGA, A.; ACS, Z. Geographical Spillovers and University Research: A Spatial Econometric Perspective. Growth and Change v. 31, p. 501-515, 2000.

AUDRETSCH, D. B., FELDMAN, M. P. R\&D spillovers and the geography of innovation and production. The American Economic Review, v. 86, p. 630-640, 1996.

BALLAND, P. A.; BOSCHMA, R.; FRENEN, K. Proximity and Innovation: From Statics to Dynamics, Regional Studies, v. 49(6), 2015.

BELUSSI, F., CALDARI, K. At the Origin of the Industrial District: Alfred Marshall and the Cambridge School." Cambridge Journal of Economics v. 33(2), p. 335-355, 2009.

BERNARDES, A.; ALBUQUERQUE, E. Cross-Over, thresholds and the interactions between Science and technology: lessons for less-developed countries. Research Policy, v. 32(5), p. 867-887.

BODAS FREITAS, I. M.; GEUNA, A.; ROSSI, F. Finding the right partners: Institutional and personal modes of governance of university-industry interactions, Research Policy, 42(1), p. 50-62, 2013.

BONACCORSI, A.; DARAIO, C. Age effects in scientific productivity The case of the Italian National Research Council (CNR) Scientometrics, v. 58(1), p. 49-90, 2003.

BORGATTI, S. P.; EVERETT, M. G.; JOHNSON, J. C. Analyzing Social Networks, London: SAGE Publications Ltd, 2013.

BOSCHMA, R. Proximity and Innovation: A Critical Assessment. Regional Studies, v. 39, 61-74, 2005.

BROEKEL, T.; BOSCHMA, R. Knowledge networks in the Dutch aviation industry: the proximity paradox Journal of Economic Geography, v. 12(2), p. 409-433, 2012.

BROEKE, T.; MUELLER, W. Critical links in knowledge networks - What about proximities and gatekeeper organizations? Industry and Innovation, v. 25(10), p. 919-939, 2018.

CAPALDO, A.; PETRUZZELLI, M. Partner Geographic and Organizational Proximity and the Innovative Performance of Knowledge-Creating Alliances European Management Review, v. 11, p. 63-84, 2014.

COHEN, W.M.; LEVINTHAL, D.A. Innovation and learning: the two faces of R\&D. The Economic Journal v. 99, p. 569-596, 1989.

COHEN, W.M.; LEVINTHAL, D.A. Absorptive capacity: a new perspective of learning and innovation. Administrative Science Quarterly, v. 35, p. 128-152, 1990. 
COHENDET, P., LLERENA, P. (1997) Learning, technical change and public policy: how to create andexploit diversity. In C. Edquist (ed.) Systems of Innovation, p. 223-241, 1997.

CRESCENZI, R.; NATHAN, M.; RODRIGUEZ-POSE, A. Do inventors talk to strangers? On proximity and collaborative knowledge creation Research Policy v. 45(1), p. 177-194, 2016.

CRESCENZI, R.; FILIPPETTI, A.; IAMMARINO, S. Academic inventors: collaboration and proximity with industry Journal of Technological Transfer, 2017.

DANELL, R. The contribution of authors: A study of the relationship between the size and composition of author teams Ed Noyons, Leiden, Netherlands: Leiden university, p. 123-13, 2014.

D'ESTE, P.; GUY, F.; IAMMATINO, S. Shaping the formation of universtiy-industry research collaboration: what type of proximity does really matter? Journal of Economic Geography, v. 13 p. 537-558, 2012.

D'ESTE, P.; PATEL, P. University-industry linkages in the UK: What are the factors underlying the variety of interactions with industry? Rsearch Policy v. 36(9), 2007.

FERNÁNDEZ, A.; FERRÁNDIZ, E.; LEÓN, M. D. Proximity dimensions and scientific collaboration among academic institutions in Europe: The closer, the better? Scientometrics, v. 106(3), p. 1073-1092, 2016.

GARCIA, R.; ARAÚJO, V.; MASCARINI, S.; SANTOS E. G.; COSTA, A. R. How the Benefits, Results and Barriers of Collaboration Affect University Engagement with Industry. Science and Public Policy, p. 347-357, v. 46(3), 2019.

GEISLER, E.; RUBENSTEIN, A. H. University-Industry Relations: A Review of Major Issues Cooperative Research and Development: The Industry-University-Government Relationship p. 43-62, 1989.

HERINGA, P.; HESSELS, L. K.; VAN DER ZOUWEN The influence of proximity dimensions on international research collaboration: an analysis of European water projects Industry and Innovation, v. 23(8), p. 753-772, 2016.

HERINGA, P.; HORLINGS, E.; VAN DER ZOUWEN, M.; VAN DEN BESSELAAR, P.; VAN VIERSSEN W. How dimensions of proximity relate to the outcome of collaboration? A survey of knowledge-intensive networks in the Dutch water sector Economics of Innovation and New Technology, v.23(7), p. 689-716, 2014.

HILBE, Joseph M. Negative binomial regression. Cambridge University Press, 2011.

HONG, W.; SU, Y. S. The effect of institutional proximity in non-local university-industry collaborations: An analysis based on Chinese patent data Research Policy v. 42, p. 454-464, 2013.

JENSEN, M. B.; JOHNSON, B.; LORENZ, E.; LUNDCALL, B.A. Forms of knowledge and modes of innovation. Research Policy v. 36, p. 680-693, 2007.

KAYGALAK, I.; REID, N. Innovation and knowledge spillovers in Turkey: The role of geographic and organizational proximity: Innovation and knowledge spillovers in Turkey Regional Science Policy \& Practice v. 8(1-2), p. 45-60, 2016. 
KIRAT. T, LUNG, Y. Innovation and proximity. Territories as loci of collective learning processes, European Urban and Regional Studies v. 6, p. 27-38, 1999.

LAGENDIJK, A.; LORENTZEN, A. Proximity, Knowledge and Innovation in Peripheral Regions. On the Intersection between Geographical and Organizational Proximity European Planning Studies, v. 15(4), 2007.

LATA, R.; PROFF, S.; BRENNER, T. The influence of distance types on co-patenting and copublishing in the USA and Europe over time. The Annuals of Regional Science. v. 61 (1), Heidelberg, 2018.

LAURSEN, K.; SALTER, A. Searching high and low: what types of firms use universities as a source of innovation? Research Policy v. 33, p. 1201-1215, 2004.

MARSHAL, A. Principles of Economics: An Introductory Volume, Londres: Macmillan, 1920.

MENA-CHALCO, J. P.; DIGIAMPIETRI, L. A.; LOPES, F. M.; CESAR JR R. M. Brazilian Bibliometric Coauthorship Networks. Journal of the Association for Information Science and Technology, v. 65(7), p. 1424-1445, 2014.

MUSCIO, A.; POZZALI, A. The effects of cultural distance in university-industry collaborations. Some evidence from Italian universities The Journal of Technology Transfer v. 38(4), 2013.

NOOTEBOOM, B. Learning and Innovation in Organizations and Economies. Oxford: Oxford University Press, 2000.

NOOTEBOOM, B., VAN HAVERBEKE, W., DUYSTERS, G., GILSING, V., VAN DEN OORD, A. Optimal cognitive distance and absorptive capacity. Research Policy, v. 36, p. 1016-1034, 2007

PACI, R.; MARROCU, E.; USAI, S. The Complementary Effects of Proximity Dimensions on Knowledge Spillovers Spatial Economic Analysis v. 9(1), p. 9-30, 2014.

PONDS, R.; OORT, F. V.; FRENKEN, K. The geographical and institutional proximity of research collaboration Regional Science, v. 86(3), p. 423-443, 2007.

SAXENIAN, A. Silicon Valley's New Immigrant Entrepreneurs, Public Policy Institute of California, 1999.

SORENSON, O.; RIVKIN, J. W.; FLEMING, L. Complexity, networks and knowledge flow Research Policy v. 35 p. 994-1017, 2006.

SMITH, C; SOTALA, K. Knowledge, networks and nations Global scientific collaboration in the 21st century The Royal Society v. 03/11, 2011. 